\title{
Surface plasmons on Pd(110): An ab initio calculation
}

\author{
U. Muniain $\odot,{ }^{1}$ R. Esteban,,${ }^{1,2,3}$ I. P. Chernov, ${ }^{4}$ J. Aizpurua, ${ }^{1,2}$ and V. M. Silkin ${ }^{1,3,5}$ \\ ${ }^{1}$ Donostia International Physics Center, P. de Manuel Lardizabal 4, 20018 San Sebastián/Donostia, Basque Country, Spain \\ ${ }^{2}$ Centro de Física de Materiales, Centro Mixto CSIC-UPV/EHU, P. de Manuel Lardizabal, 5, 20018 San Sebastián/Donostia, \\ Basque Country, Spain \\ ${ }^{3}$ IKERBASQUE, Basque Foundation for Science, 48009 Bilbao, Basque Country, Spain \\ ${ }^{4}$ Ingineering School of Nuclear Technology, Tomsk Polytechnic University, pr. Lenina 30, 634050 Tomsk, Russia \\ ${ }^{5}$ Departamento de Polímeros y Materiales Avanzados: Física, Química y Tecnología, Facultad de Ciencias Químicas, \\ Universidad del País Vasco UPV/EHU, Apartado 1072, 20080 San Sebastián/Donostia, Basque Country, Spain
}

(Received 27 October 2020; accepted 14 December 2020; published 8 January 2021)

\begin{abstract}
The surface excitation spectra of the $\operatorname{Pd}(110)$ surface is analyzed in the framework of the time-dependent density functional theory. The $a b$ initio electronic structure of this surface is included into the evaluation of the surface response function using a linear response approach. At small momentum transfer the extrapolated energy of the surface plasmon is close to electron-energy-loss-spectroscopy measurements and can be understood considering the bulk Pd dielectric function. However, upon momentum increase the evaluated dispersion of the surface plasmon presents significant discrepancies with the published experimental data. While the experimental surface plasmon dispersion initially presents a strong negative slope, our calculations indicate a weak positive dispersion at all finite momentum transfers where this collective excitation is observed. Interestingly, we do not observe any noticeable effect on the collective surface excitations induced by the large number of surface states in the valence band of $\mathrm{Pd}(110)$. These states do not produce any additional mode at energies below the surface plasmon.
\end{abstract}

DOI: 10.1103/PhysRevB.103.045407

\section{INTRODUCTION}

A crucial characteristic of an electronic system in any material is its collective behavior in response to an applied electric field. Its most relevant manifestation in infinite solids is the collective charge density oscillations called plasmons $[1,2]$. The energy of these bulk plasmonic modes in metals, $\omega_{\mathrm{P}}$, is usually in the ultraviolet spectral range and, for a simple free-electron gas (FEG) model, it is determined by the average valence electron density.

Additional modes can be generated by truncating the solid. For instance, at the plane interface between a metal and a vacuum an additional collective excitation, the so-called surface plasmon (SP), appears [3]. In the FEG model, the energy of this mode, $\omega_{\mathrm{SP}}$, in the long-wavelength limit is linked to $\omega_{\mathrm{P}}$ by the simple relation $\omega_{\mathrm{SP}}=\omega_{\mathrm{P}} / \sqrt{2}$.

The properties of surface plasmons have been the subject of great attention from the experimental and theoretical sides for more than six decades [4-10]. Notably, many works were devoted to establishing its energy dispersion as a function of the in-plane momentum transfer $\mathbf{q}_{\|}$. Theories based on the FEG model predicted that in metals such dispersion should initially present a negative slope with increasing $q_{\|}=\left|\mathbf{q}_{\|}\right|$ (denoted negative dispersion in the following for short), reach a minimum at a certain $q_{\|}$, and become positive at larger $q_{\|}$. Several experiments confirmed this picture in simple metals like alkalies, aluminium, magnesium, etc. [11,12].

However, in the course of that work it was realized that even for such simple metals, a proper theoretical description of the surface plasmon properties should go beyond the simplistic FEG model. For instance, the experimental $\omega_{\text {SP }}$ systematically deviates from the FEG predictions. Moreover the surface plasmon dispersion may deviate qualitatively from the one predicted by the FEG. A remarkable example of this is the surface of silver. The experimentally measured surface plasmon dispersion [13-18] was found to be positive, contrary to the FEG model prediction. This discrepancy was attributed to the absence of the totally occupied $4 d$ valence bands on the FEG-based theories [19-22]. The same conclusion was reached in a study based on a Ag model potential used to describe its surface electronic structure [23]. Another serious problem was found in the description of the lifetime of the surface plasmons. The FEG model predicts that at $q_{\|}=0$ the lifetime of a surface plasmon should be infinite, i.e., the surface-plasmon-peak width in the loss spectra should be zero. However, in the experiments even at small $q_{\|}$the measured linewidth is finite.

The absence of band structure effects in the FEG models can thus lead to incorrect predictions. Indeed, in real solids the electronic structure deviates from the free-electron behavior. As a result, the intra- and interband electron transitions may disturb the electronic excitation spectra both in the bulk and at the surface. The development of modern band structure calculation methods has allowed for a proper inclusion of the electronic band structure in the evaluation of electronic excitation spectra. In the case of surfaces of solids a first successful realization of such approach considered the $\operatorname{Mg}(0001)$ surface [24]. It was demonstrated that the inclusion of the realistic 
surface electronic structure obtained in the $a b$ initio calculation as well as of the proper dynamical exchange-correlation effects allows us to reproduce the experimental dispersion of the surface plasmon energy and linewidth remarkably well. Later on, similar calculations on the basis of the $a b$ initio surface electronic structure were realized for a number of clean and adsorbate-covered surfaces. In general, it has been demonstrated that including the surface electronic structure is a key ingredient that considerably improves agreement with the experimental findings. Significantly, when the Ag valence $d$ electrons were properly taken into account in the $a b$ initio time-dependent density functional theory (TDDFT) calculations, a positive surface plasmon dispersion in agreement with the experiment is recovered [25-27]. A similar situation occurs in the case of a gold surface plasmon [28], i.e., a measured positive dispersion [28-30] is reproduced by the $a b$ initio calculations [26].

The aforemetioned works indicate that the current $a b$ initio descriptions in the framework of TDDFT are able to reproduce the experimentally determined properties of surface plasmons in many materials, but there are still systems where an agreement is not straightforward because of the complexity of their electronic structure. Especially, in $d$ metals such as palladium, numerous inter-band transitions with different probability can disturb the excitation spectra in a significant way, which complicates the theoretical description and could potentially lead to new effects.

The surface of palladium was intensively studied experimentally with electron energy loss spectroscopy (EELS) in the past. Surface excitations on single-crystal surfaces with energies between 6.8 and $7.0 \mathrm{eV}$ were observed [31-35]. The surface plasmon energy of polycrystalline Pd was measured at an energy of $6.5 \mathrm{eV}[36,37]$. In other scattering experiments [38-41] energy-loss peaks in the 7.2-7.5 eV energy range, which might also be related to the surface plasmon, were detected as well. A nondispersing surface plasmon peak with energy at $6.6 \mathrm{eV}$ was measured on $\mathrm{Pd}(111)$ by Netzer and $\mathrm{El}$ Gomati [42]. In contrast, Rocca et al. $[43,44]$ found that the surface plasmon in $\mathrm{Pd}(110)$ shows an unusually strong negative linear initial dispersion with transferred momentum. They pointed out that $\operatorname{Pd}(110)$ behaves in this respect differently in comparison to noble and simple metals. However, a detailed analysis of such anomalous dispersion is still pending.

Motivated by the Rocca et al. findings $[43,44]$, in this paper we calculate the dispersion and damping of the surface plasmon on $\operatorname{Pd}(110)$ employing an ab initio aproach [24] and compare it with existing experimental data. A second motivation is to investigate in detail the excitation spectra of $\operatorname{Pd}(110)$ at low energies. It was recently demonstrated that in bulk Pd the presence of several groups of carriers at the Fermi level with different group Fermi velocities led to the appearance of acoustic plasmons characterized by a soundlike dispersion at energies below $\approx 1 \mathrm{eV}[45,46]$. Here we analyze whether such remarkable bulk modes have any influence in the surface excitation spectra. Furthermore, the Pd surfaces present several surface states in the vicinity of the Fermi level [47-49]. We investigate whether their existence can lead to the realization of an additional low-energy surface mode - an acoustic surface plasmon [50] - observed on other surfaces supporting surface electronic states [30,51-58].
The paper is organized as follows. A brief description of the calculation methods used and computational details are presented in Sec. II. The calculated electronic structure and excitation spectra of the $\operatorname{Pd}(110)$ surface along with the comparison with experiments are reported in Sec. III. The summary and conclusions are given in Sec. IV. Unless otherwise stated explicitly, atomic units are employed throughout, i.e., $e^{2}=\hbar=m_{e}=1$.

\section{CALCULATION METHODS}

We calculate the response function of the $\operatorname{Pd}(110)$ surface employing a finite-thickness-layer geometry. The information about the dynamical electronic excitations supported by this system and characterized by in-plane momentum $\mathbf{q}_{\|}$and energy $\omega$ can be obtained from the imaginary part of the surface response function $g$, called surface loss function. This quantity can be directly compared with the spectra measured in the EELS experiments realized in reflection geometry [59]. The peaks in the calculated surface loss function determine the energy and lifetime of the surface collective excitations. The $g$ function is expressed as [60]

$$
g\left(\mathbf{q}_{\|}, \omega\right)=\int d \mathbf{r} e^{q_{\|} \cdot z} n^{\text {ind }}\left(\mathbf{r}, \mathbf{q}_{\|}, \omega\right),
$$

where the $x-y$ plane is parallel to the surface, $z$ is the coordinate normal to the surface, i.e., $\mathbf{r} \equiv\{x, y, z\} \equiv\left\{\mathbf{r}_{\|}, z\right\}$, and the solid boundary is located at $z=0$. In Eq. (1) $n^{\text {ind }}\left(\mathbf{r}, \mathbf{q}_{\|}, \omega\right)$ is the surface charge density induced by an external potential of the form $V^{\text {ext }}\left(\mathbf{r}, \mathbf{q}_{\|}, \omega\right)=-\left(2 \pi / q_{\|}\right) \exp \left[q_{\|} z+\mathrm{i}\left(\mathbf{q}_{\|} \mathbf{r}_{\|}-\omega t\right)\right]$. In the framework of TDDFT $[61,62]$, the induced density is determined as

$$
n^{\text {ind }}\left(\mathbf{r}, \mathbf{q}_{\|}, \omega\right)=\int d \mathbf{r}^{\prime} \chi\left(\mathbf{r}, \mathbf{r}^{\prime}, \omega\right) V^{\mathrm{ext}}\left(\mathbf{r}^{\prime}, \mathbf{q}_{\|}, \omega\right) .
$$

where $\chi\left(\mathbf{r}, \mathbf{r}^{\prime}, \omega\right)$ is the density response function of an interacting electron system. In TDDFT, this quantity is related to the response function of the noninteracting Kohn-Sham electrons $\chi^{o}$ through the integral equation $\chi=\chi^{o}+\chi^{o}\left(v_{c}+\right.$ $\left.K_{\mathrm{xc}}\right) \chi$. Here $v_{c}$ is the bare Coulomb potential and $K_{\mathrm{xc}}$ accounts for the dynamical exchange-correlation (XC) effects. For the latter, two approximations are used in this work: (i) the random-phase approximation (RPA) in which $K_{\mathrm{xc}}$ is set to zero and (ii) the adiabatic local density approximation (ALDA) [62]. Previous studies showed that the XC effects have little impact on the excitation spectra at small $q_{\|}$'s $[12,24,63,64]$. Upon momentum increase, however, the role of $\mathrm{XC}$ effects is gradually gaining importance and ALDA gives a better agreement [12,20,24,65,66] with experiments. The XC effects are smaller in systems characterized by high valence charge density, which is the case of Pd. Therefore, as will be discussed in the following, the inclusion of $K_{\mathrm{xc}}$ within ALDA has no significant impact on the properties of the surface plasmon of Pd at any $\mathbf{q}_{\|}$except its linewidth at large $q_{\|}$.

In order to evaluate $\chi^{o}$, we obtain the Kohn-Sham electronic structure of the $\operatorname{Pd}(110)$ surface by employing a geometry of slabs periodically repeated in the $z$ direction. The experimental value [67] $a=7.3512$ a.u. for the facecentered-cubic lattice parameter has been chosen in this work. Slabs with 19 atomic layers simulating the $\operatorname{Pd}(110)$ surface 
are employed. These slabs are separated by a vacuum equivalent to nine interlayer spacings. In the low-energy electron diffraction experiment, a surface relaxation for the top three layers $\delta_{12}=-5.1 \pm 1.5 \%$ and $\delta_{23}=+2.9 \pm 1.5 \%$ was reported [68]. We have verified (not shown) that such relaxation does not affect visibly neither the electronic structure nor the excitation spectra of $\operatorname{Pd}(110)$, and therefore it is not included in the results below.

The self-consistent band structure was obtained employing a homemade code [69]. The Troullier-Martins normconserving pseudopotential was used for the description of the electron-ion interaction in Pd [70]. The Ceperley-Alder form for the XC potential was chosen [71]. The repeated slab geometry employed here allows us to transform the quantities defined in the real space into the matrices of their Fourier transformations. The matrix elements of the noninteracting response function can be determined according to

$$
\begin{aligned}
\chi_{\mathbf{G G}^{\prime}}^{o}\left(\mathbf{q}_{\|}, \omega\right)= & \frac{2}{S} \sum_{\mathbf{k}_{\|}}^{\text {SBZ }} \sum_{n}^{\text {occ unocc }} \sum_{n^{\prime}} \frac{f_{n \mathbf{k}_{\|}}-f_{n^{\prime}} \mathbf{k}_{\|}+\mathbf{q}_{\|}}{E_{n \mathbf{k}_{\|}}-E_{n^{\prime} \mathbf{k}_{\|}+\mathbf{q}_{\|}}+(\omega+\mathrm{i} \eta)} \\
& \times\left\langle\phi_{n \mathbf{k}_{\|}}\left|e^{-\mathrm{i}\left(\mathbf{q}_{\|}+\mathbf{G}\right) \mathbf{r}}\right| \phi_{n^{\prime}} \mathbf{k}_{\|}+\mathbf{q}_{\|}\right\rangle \\
& \times\left\langle\phi_{n^{\prime} \mathbf{k}_{\|}+\mathbf{q}_{\|}}\left|e^{\mathrm{i}\left(\mathbf{q}_{\|}+\mathbf{G}^{\prime}\right) \mathbf{r}}\right| \phi_{n \mathbf{k}_{\|}}\right\rangle
\end{aligned}
$$

where the factor 2 accounts for the spin, $S$ is the in-plane unit cell area, and $n$ and $n^{\prime}$ are the band indices of the occupied (occ) and unoccupied (unocc) states, respectively. The summation over the wave vector $\mathbf{k}_{\|}$is realized in the surface Brillouin zone (SBZ). The Fermi occupation factors $f_{n \mathbf{k}_{\|}}$'s are calculated at a temperature of $10 \mathrm{meV}$ in order to reduce the noise caused by the discrete summation in $\mathbf{k}_{\|} . E_{n \mathbf{k}_{\|}}$ and $\phi_{n \mathbf{k}_{\|}}$are the Kohn-Sham energies and wave functions, respectively. Formally, $\eta$ in Eq. (3) is infinitesimal, but in practical calculations one should use some finite value which introduces an artificial broadening in the calculated spectra. In order to eliminate such broadening, we use an equivalent formulation where, first, the imaginary part of $\chi_{\mathbf{G G}^{\prime}}^{o}$ is evaluated as described, e.g., in Ref. [72]. Subsequently, $\operatorname{Re}\left[\chi_{\mathbf{G G}^{\prime}}^{o}\right]$ is calculated via the Kramers-Kronig relation. This alternative procedure accelerates several times the surface response calculations.

The final expression $[24,59]$ for the surface response function employed here is

$g\left(\mathbf{q}_{\|}, \omega\right)=-\frac{2 \pi}{q_{\|}} \int d z \int d z^{\prime} \chi_{\mathbf{G}_{\|}=\mathbf{G}_{\|}^{\prime}=0}\left(z, z^{\prime}, \mathbf{q}_{\|}, \omega\right) e^{q_{\|} \cdot\left(z+z^{\prime}\right)}$.

In principle, all three-dimensional local-field effects are included in the evaluation of $g$ through the integral equation relating $\chi$ and $\chi^{o}$. However, since the local-field effects play little role in $\mathrm{Pd}$ at moderate momentum transfers [73] we expand $\chi_{\mathbf{G G}^{\prime}}^{o}$, and thus $\chi_{\mathbf{G G}^{\prime}}$, over the $\mathbf{G}$ vectors directed in the $z$ direction, i.e., only the local-field effects in the direction perpendicular to the surface are taken into account. In the present calculations we used 151 such $\mathbf{G}$ vectors in the expansion of the $\chi^{o}$ matrices. The calculation of $\chi_{\mathbf{G G}^{\prime}}^{o}(\mathbf{q}, \omega)$ was realized using our code [74]. Summation over the SBZ was performed using a $136 \times 96$ grid of $\mathbf{k}_{\|}$wave vectors. Since the $\mathbf{k}_{\|}$and the $\mathbf{q}_{\|}+\mathbf{k}_{\|}$vectors belong to the same mesh, such a grid imposes a minimum value $q_{\|}^{\min }=\frac{2 \pi}{a \cdot 96}=0.017 \AA^{-1}$ for $\mathbf{q}_{\|}$'s accessible in our calculations. We included all the Pd valence states with energies up to $25 \mathrm{eV}$ above the Fermi level. In order to avoid the coupling between slabs we follow the procedure proposed by Nazarov [59].

\section{RESULTS AND DISCUSSION}

\section{A. $\operatorname{Pd}(110)$ electronic band structure}

The calculated electronic structure for the 19-atomic layer $\operatorname{Pd}(110)$ slab is reported in Fig. 1. One can see that in palladium, the $4 d$ electronic bands completely dominate the occupied and unoccupied valence band structure around the Fermi level. The $s-p$ states are strongly hybridized with these states in the energy interval between -5.5 and $0.5 \mathrm{eV}$. A wide energy gap for the bulk-like states in Fig. 1 is located in the -0.2 to $2.2 \mathrm{eV}$ interval around the $\overline{X S}$ and $\overline{S Y}$ symmetry directions. Additionally, above the Fermi level we find two energy gaps around the $\bar{X}$ and $\bar{Y}$ points with the bottom at energies of 3.4 and $1.1 \mathrm{eV}$, respectively. Also in the occupied valence band structure of Fig. 1 some other narrow gaps can be detected.

In the surface electronic structure reported in Fig. 1 the surface states are highlighted by blue dots. In the unoccupied part we find at the bottom of an energy gap a surface state with minimum energy at $1.3 \mathrm{eV}$ at $\bar{Y}$. Its presence correlates rather well with inverse photoemission experiment [49]. In the same energy gap we observe a second surface state with energy of $3.0 \mathrm{eV}$ at $\bar{Y}$. In the experiment [49] this surface state is located at $3.5 \mathrm{eV}$ and its dispersion is almost flat whereas in the calculation it has a pronounced paraboliclike dispersion. The origin of such discrepancy is not clear. Further, around the $\bar{\Gamma}$ point we find a couple of energy-split bands above $4 \mathrm{eV}$ with free-electron-like dispersion. Such states may be considered as precursors of the resonance image potential states, detected at $3.8 \mathrm{eV}$ in $\bar{\Gamma}$ in the experiment [49].

In the vicinity of the Fermi level we find a surface state of $d$ type characterized by small dispersion and unoccupied at the $\bar{Y}$ point. Upon moving from this point towards $\overline{\mathrm{S}}$ this surface state band crosses the Fermi level and quickly becomes occupied. On the contrary, along $\overline{\mathrm{Y} \Gamma}$ this surface state presents a positive dispersion and remains above the Fermi level. Such a state was observed experimentally [49] and found in previous $a b$ initio calculations $[47,48]$. We find a similar surface state crossing the Fermi level around the $\bar{S}$ point. Its energy position and dispersion is in agreement with other calculations [48], but this wave-vector region was not probed in the aforementioned experiment [49]. The existence of surface states crossing the Fermi level suggests a scenario where the out-of-phase collective charge oscillation involving such states and bulk electronic system might result in a surface collective excitation with soundlike dispersion, the so-called acoustic surface plasmon $[50,75]$. We analyze such a possibility in Sec. III B.

Below the Fermi level in the electronic structure of Fig. 1 we find several surface states and surface resonances of mainly $d$ type. Their existence may result in numerous interband transitions in the surface region. However, such a rich surface electronic structure in $\operatorname{Pd}(110)$ does not allow us to 


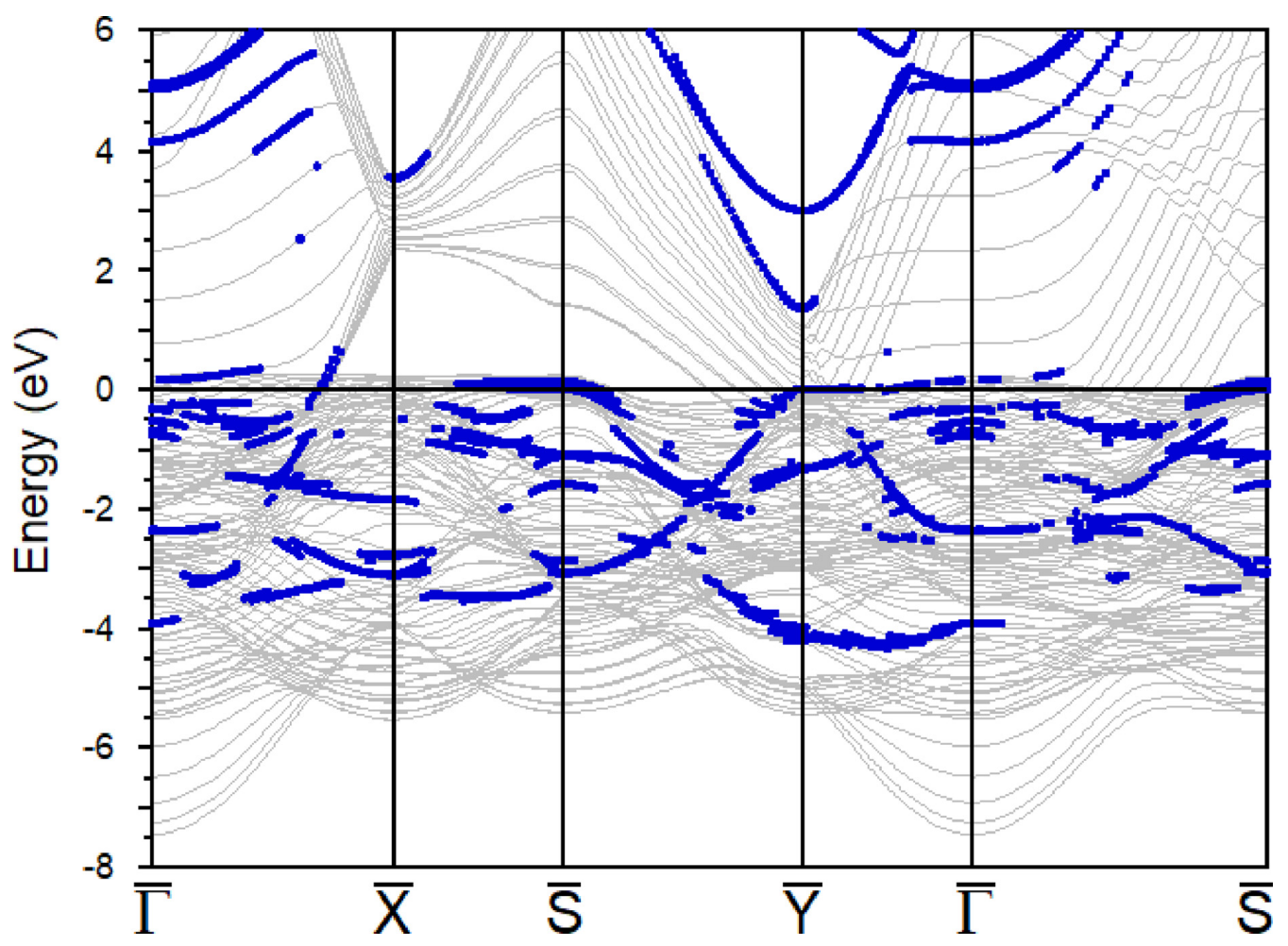

FIG. 1. Band structure of the 19-atomic layer Pd(110) film calculated along the lines of high symmetry in the surface Brillouin zone. The states with strong localization in the surface atomic layer are highlighted by blue dots.

establish a specific role played by each of these states in the formation of surface excitation spectra.

\section{B. Pd(110) surface excitation spectrum}

In Fig. 2 we report the ALDA surface loss function $\operatorname{Im}\left[g\left(\mathbf{q}_{\|}, \omega\right)\right]$ at momentum transfers $\mathbf{q}_{\|}$along two symmetry directions, $\overline{\Gamma X}$ and $\overline{\Gamma Y}$, of the SBZ. In general, the calculated spectrum is very similar for both directions indicating a remarkable isotropic behavior. One can see that the surface loss function is dominated by a rather wide surface plasmon peak labeled as SP (yellow-white color in the contour plot of Fig. 2). Over a large portion of momentum transfer values (for $q_{\|}>0.1 \AA^{-1}$ ), this peak shows up at energies in the $7.3-7.6 \mathrm{eV}$ range. For values of $q_{\|}<0.1 \AA^{-1}$, the peak in the surface loss function is shifted, since the interaction between the surfaces of the slab is noticeable for such small $q_{\|}$values. Therefore, one can obtain the value of the semi-infinite surface plasmon $\omega_{\mathrm{SP}}\left(\mathbf{q}_{\|}=0\right)$, by direct extrapolation of the loss function from the values in the $q_{\|} \approx 0.1-0.4 \AA^{-1}$ range in Fig. 2, which gives a value of $\omega_{\mathrm{SP}}\left(\mathbf{q}_{\|}=0\right)=7.35 \mathrm{eV}$. This value is consistent with the one obtained from the surface loss function with the use of a bulk dielectric function of Pd [76,77] and also in good agreement with the experimentally determined value of $7.37 \pm 0.10 \mathrm{eV}$, obtained in Ref. [43].

The surface plasmon dispersion in Fig. 2 is severely distorted at momentum transfers below a certain critical value, with the energy of the plasmonic peak shifting towards smaller energy as $\mathbf{q}_{\|} \rightarrow 0$. This behavior is due to the finite thickness of the slab geometry considered in the simulations, as can be confirmed by plotting in Fig. 2 the classical dispersion of sur- face hybrid plasmons in a thin layer (dashed lines). The latter are obtained from a thin-film Drude model [3,5] where the surface plasmon frequency $\omega_{\mathrm{SP}}\left(\mathbf{q}_{\|}=0\right)$ is set to the extrapolated value of $7.35 \mathrm{eV}$. The interaction between the plasmon modes supported at the two metal-dielectric interface surfaces leads to two dispersion branches corresponding to two different hybrid surface modes, the symmetric plasmon $\mathrm{SP}^{-}$at lower energies and the antisymmetric plasmon $\mathrm{SP}^{+}$at larger energies [3,5]. The classical dispersion of the symmetric $\mathrm{SP}^{-}$ mode clearly follows a similar tendency at small $\mathbf{q}_{\|}$as the peak of the loss function obtained within TDDFT. However, the peak energy obtained from the TDDFT calculations shows a higher energy value in comparison to the prediction of the Drude model (dashed lines). We understand such behavior as a consequence of the effect of interband transitions involving the $d$ valence states, which are not included in the reference result within the Drude model. It is actually known that even in a relatively simple metal such as lead, the band structure effects are sufficiently large to disturb the surface excitation spectra [78].

Regarding the upper-energy antisymmetric $\mathrm{SP}^{+}$surface mode, only a very weak broad feature can be discerned in the TDDFT calculations at the smallest $q_{\|}$above $7.35 \mathrm{eV}$ (hardly distinguishable in Fig. 2), which suggests that the intensity of this mode is strongly suppressed by the interband transitions. Such an explanation is backed up by the results in Fig. 3(a) where we report $\operatorname{Im}\left[g\left(\mathbf{q}_{\|}, \omega\right)\right]$ in the region of low momentum transfers as obtained from a classical calculation that uses the experimental bulk dielectric function of palladium [79]. The $\mathrm{SP}^{-}$hybrid mode dominates in this momentum range, while the intensity of the upper-energy $\mathrm{SP}^{+}$peak is negligible 


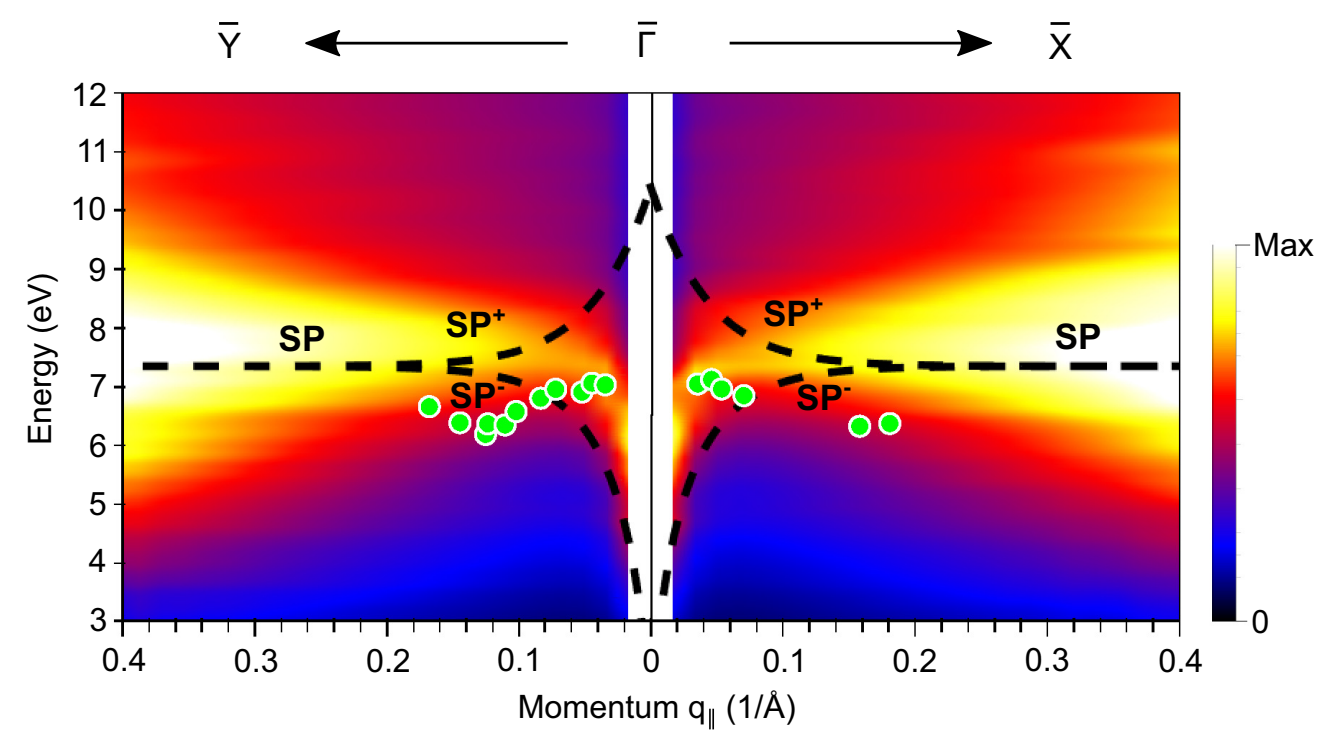

FIG. 2. ALDA surface loss function of Pd(110) calculated for the 19-atomic layer slab along the lines of high symmetry, $\overline{\Gamma X}$ and $\overline{\Gamma Y}$, in the surface Brillouin zone. The dashed lines show the classical dispersion relationship for surface modes in a thin film, where the surface plasmon SP splits into two hybrid surface modes $\mathrm{SP}^{-}$and $\mathrm{SP}^{+}$for small momentum transfers. This dispersion has been obtained for a film of the same thickness as in the ab initio calculations and using a Drude model to describe the permittivity of Pd, where the Drude surface plasmon frequency $\omega_{\mathrm{SP}}=7.35 \mathrm{eV}$ is obtained from the extrapolation of the TDDFT results to $q_{\|}=0$. The experimental data points indicated by green dots represent the measured SP dispersion, according to Ref. [43].

(in contrast, a clear $\mathrm{SP}^{+}$peak appears for the corresponding calculations using Drude permittivity, not shown). Moreover, a strong deviation in the dispersion of both modes in comparison with the Drude model (dashed lines) is also obtained. Notably, these classical results using the experimental bulk dielectric function are quite similar to those obtained from the $a b$ initio calculations [zoom to the low- $q_{\|}$ab initio results shown in Fig. 3(b)] and confirm the strong influence of the interband transitions on the low $-q_{\|}$region of the dispersion.

We focus next on the dispersion of the SP at larger values of $\mathbf{q}_{\|}$. In this regime, thin-film effects are much weaker, and the results can be directly interpreted as being very close to those of a semi-infinite substrate. As discussed previously, at small $\mathbf{q}_{\|}$the loss function shows a peak at around $7.35 \mathrm{eV}$ that is in good agreement with experimental results [43]. However, the calculated surface plasmon dispersion presents a weak uprise with momentum increase up to the maximum $q_{\|}$value explored in our calculations, in striking disagreement with the experimental results, indicated by the green circles in Fig. 2. In the experiment the $\omega_{\mathrm{SP}}\left(\mathbf{q}_{\|}\right)$dispersion is negative up to $0.16 \AA^{-1}$ along the $\overline{\Gamma X}$ direction, and to $0.12 \AA^{-1}$ along the $\overline{\Gamma Y}$ one. Moreover, the slope is unusually large [43]. At
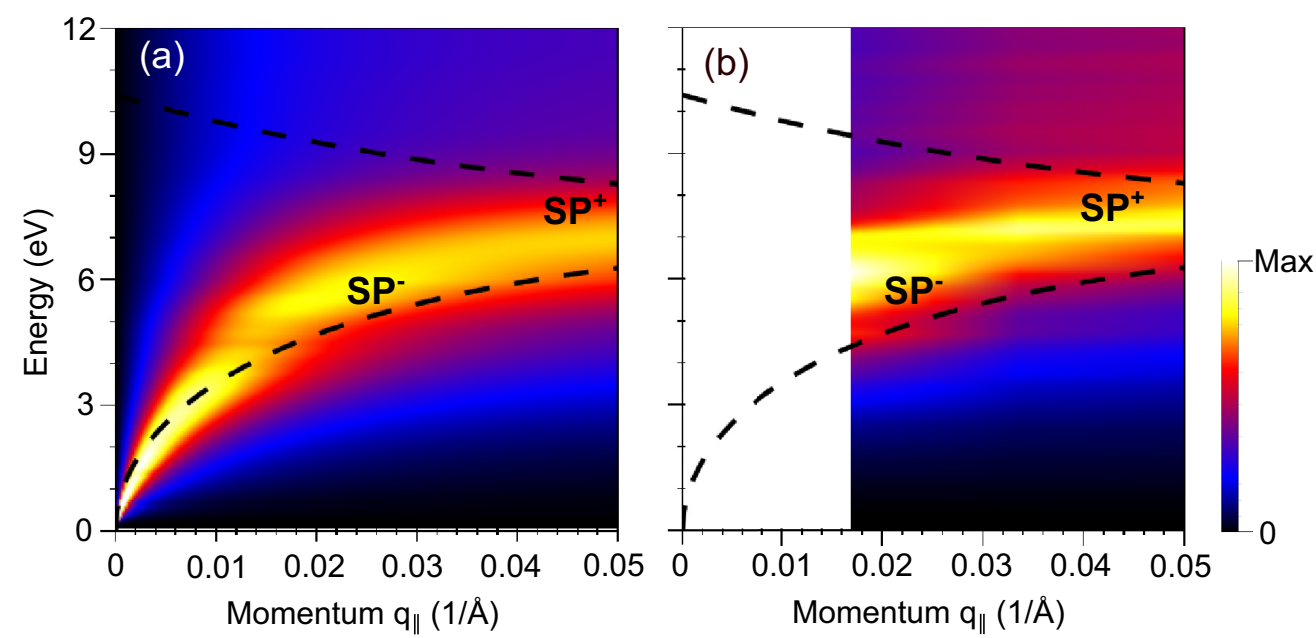

FIG. 3. (a) Surface loss function of a 19-atomic layer $\operatorname{Pd}(110)$ slab as obtained with a classical electromagnetic calculation using the experimental bulk dielectric function of Pd [79]. (b) Surface loss function of $\operatorname{Pd}(110)$ calculated within the $a b$ initio approach along the $\overline{\Gamma \bar{X}}$ line (zoom in of Fig. 2). The dashed lines show the dispersion of the two hybrid surface modes $\mathrm{SP}^{-}$and $\mathrm{SP}^{+}$according to the Drude model as in Fig. 2. The blanked region in (b) as well as in Fig. 2 at $q_{\|}<0.017 \AA^{-1}$ is inaccessible in our ab initio calculations. 


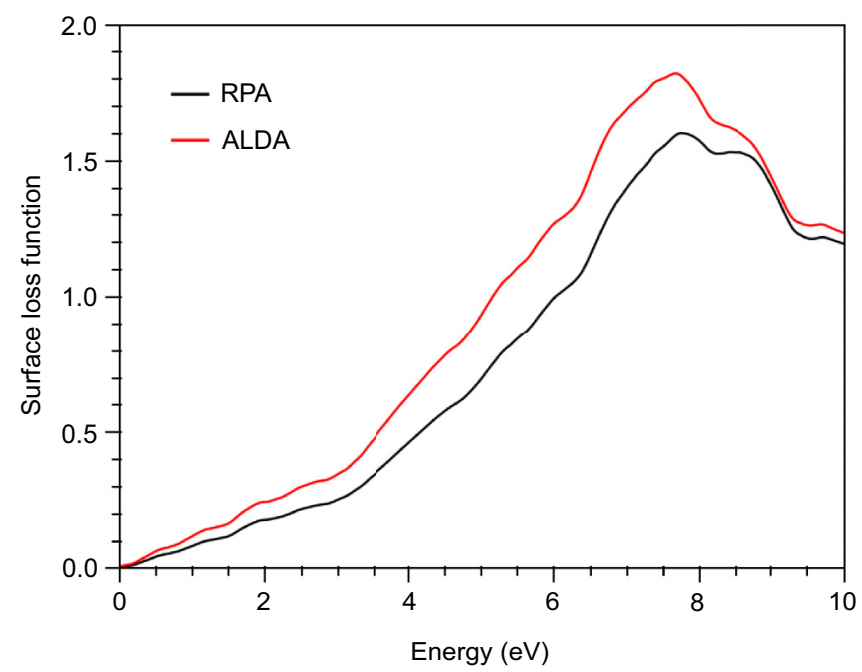

FIG. 4. The RPA and ALDA surface loss functions of Pd(110) calculated at $q_{\|}=0.370 \AA^{-1}$.

larger momentum transfers the dispersion becomes positive (up to the maximum collected experimental value of 0.18 $\AA^{-1}$ ). On the other hand, in the EELS experiment realized on Pd(111) Netzer and El Gomani found [42] no dispersion for the losses assigned to the surface plasmon, similarly to our $\operatorname{Pd}(110)$ calculations. However, the measured energy of $6.6 \mathrm{eV}$ in that experiment is rather far from the result of our calculations and from more recent experimental results by Rocca et al. [43] Clearly, this issue points out the importance of band effects in the establishment of the energy and dispersion of the surface plasmon, and requires further analysis.

We compare next the ALDA results with those obtained with RPA. At small $\mathbf{q}_{\|}$the calculated surface loss function $\operatorname{Im}[g]$ is identical in both models. Upon momentum increase ALDA gives a generally larger value of $\operatorname{Im}[g]$ in comparison with RPA. This difference mainly occurs on the low-energy side of the surface plasmon peak. Further, the SP peak in ALDA is shifted towards slightly lower energies than in RPA. These effects become more pronounced upon momentum transfer increase. As seen in Fig. 4, at $q_{\|}=0.370 \AA^{-1}$ the SP peak in the ALDA curve is centered at $\omega \approx 7.6 \mathrm{eV}$ whereas in the RPA calculation it locates at $\omega \approx 7.7 \mathrm{eV}$. The difference is much smaller than the linewidth of the SP peak, which confirms the small effect of the dynamical exchange-correlations beyond RPA on the surface plasmon dispersion on $\operatorname{Pd}(110)$ in comparison, e.g., to the $\mathrm{Mg}$ surface [24].

From the calculated $\operatorname{Im}[g(\mathbf{q}, \omega)]$ we also extracted the linewidth $\Delta_{\mathrm{SP}}$ of the surface plasmon peak, which is directly connected to the losses. Relatively large values for $\Delta_{\mathrm{SP}}$ are found even at small momentum transfers, which can be explained by an efficient decay into electron-hole pairs involving the electronic states in the valence $d$ system. Indeed, at $q_{\|} \approx$ $0.05 \AA^{-1} \Delta_{\mathrm{SP}}$ is about $1.8 \mathrm{eV}$ in both RPA and ALDA. As the momentum amplitude becomes larger, the linewidth of the surface plasmon peak gradually broadens due to an increasing decay into incoherent electron-hole pairs. At $q_{\|}=$ $0.4 \AA^{-1}$ it reaches a value of $3.3 \mathrm{eV}(4.9 \mathrm{eV})$ in RPA (ALDA). A more efficient damping at finite momentum transfers for calculations that employ the ALDA kernel is in line with other calculations $[24,80,81]$. However, despite this large linewidth, the surface plasmon peak is a dominant feature in the loss spectra in $\operatorname{Pd}(110)$ at all $\mathbf{q}_{\|}$'s as seen in Fig. 2.

Our assignment of the dominating peak in the calculated surface loss function to the surface plasmon of $\operatorname{Pd}(110)$ is supported by the analysis of the charge density distribution $n^{\text {ind }}\left(z, \mathbf{q}_{\|}, \omega\right)$ induced at the surface by the external timevarying potential $V^{\text {ext }}\left(\mathbf{r}, \mathbf{q}_{\|}, \omega\right)$. In Fig. 5 we report the real and imaginary parts [panels (a) and (b), respectively] of $n^{\text {ind }}\left(z, \mathbf{q}_{\|}, \omega\right)$ evaluated at small momentum transfer $q_{\|}=$ $0.084 \AA^{-1}$ along the $\overline{\Gamma X}$ symmetry direction as a function of energy $\omega$ and of the coordinate $z$ normal to the surface. The shape of $\operatorname{Im}\left[n^{\text {ind }}\left(z, \mathbf{q}_{\|}, \omega \approx \omega_{\text {SP }}\right)\right]$ in Fig. 5(b) indicates that at energies near the peak of the loss function, $\omega_{\mathrm{SP}} \approx$ $7.35 \mathrm{eV}$, the charge density oscillations have a characteristic distribution with the dominating maximum located above the top palladium atomic layer. For the small momentum transfer considered, the induced density penetrates rather deeply into the crystal, with a slow decay of its amplitude and an oscillating behavior of the same periodicity as the crystal lattice (the atomic planes are indicated in the figure by the solid horizontal lines). On the other hand, $\operatorname{Re}\left[n^{\text {ind }}\left(z, \mathbf{q}_{\|}, \omega\right)\right]$ in Fig. 5(a) shows strong undamped oscillating behavior for energies above $\omega_{\mathrm{SP}}=7.35 \mathrm{eV}$, which indicates optical transparency of the crystal since the electronic system cannot screen efficiently the external electric field at such high frequencies.

We calculated the position of the centroid of induced density corresponding to the real part of the first moment of the induced charge density. At the surface plasmon energy of $\omega_{\mathrm{SP}}=7.35 \mathrm{eV}$ this value averaged over several small values of $q_{\|}$is 1.2 a.u. above the geometrical surface position (the latter is marked by a dashed horizontal line). Thus, the position of the centroid of charge is located outside of the surface, which resembles the result for simple metals. Intriguingly, this is contrary to the behavior of $\mathrm{Ag}$ and $\mathrm{Au}$, where it is discussed that the effect of the $d$ electrons consists in pulling the centroid of charge inside the metal [5]. On the other hand, it seems that the $d$ states in $\mathrm{Pd}$ prevent the full realization of the simple metal scenario where the initial surface plasmon dispersion is negative.

The resulting net effect of the outside location of the induced density centroid and the $d$ states results in the weak positive surface plasmon dispersion in $\operatorname{Pd}(110)$ over an extended momentum transfer range. It seems that the more delocalized $d$ states in Pd produce a less pronounced modification of the surface plasmon dispersion in comparison to $\mathrm{Ag}$ and $\mathrm{Au}$. This conclusion is based on the analysis of the peak position in the surface loss function calculated only at $q_{\|}$'s larger than about $0.05 \AA^{-1}$, since at smaller values finite-thinness-film effects do not allow us to determine the intrinsic surface plasmon dispersion. To explore the surface plasmon properties in the small $q_{\|}$region significantly thicker slabs should be considered.

We next consider the situation of large momentum transfer in the induced charge density. For illustration, in Fig. 6 we report the real and imaginary parts of $n^{\text {ind }}\left(z, \mathbf{q}_{\|}, \omega\right)$ evaluated at $q_{\|}=0.336 \AA^{-1}$ along the $\overline{\Gamma \mathrm{X}}$ symmetry direction. For this momentum transfer the region near the surface probed by the 

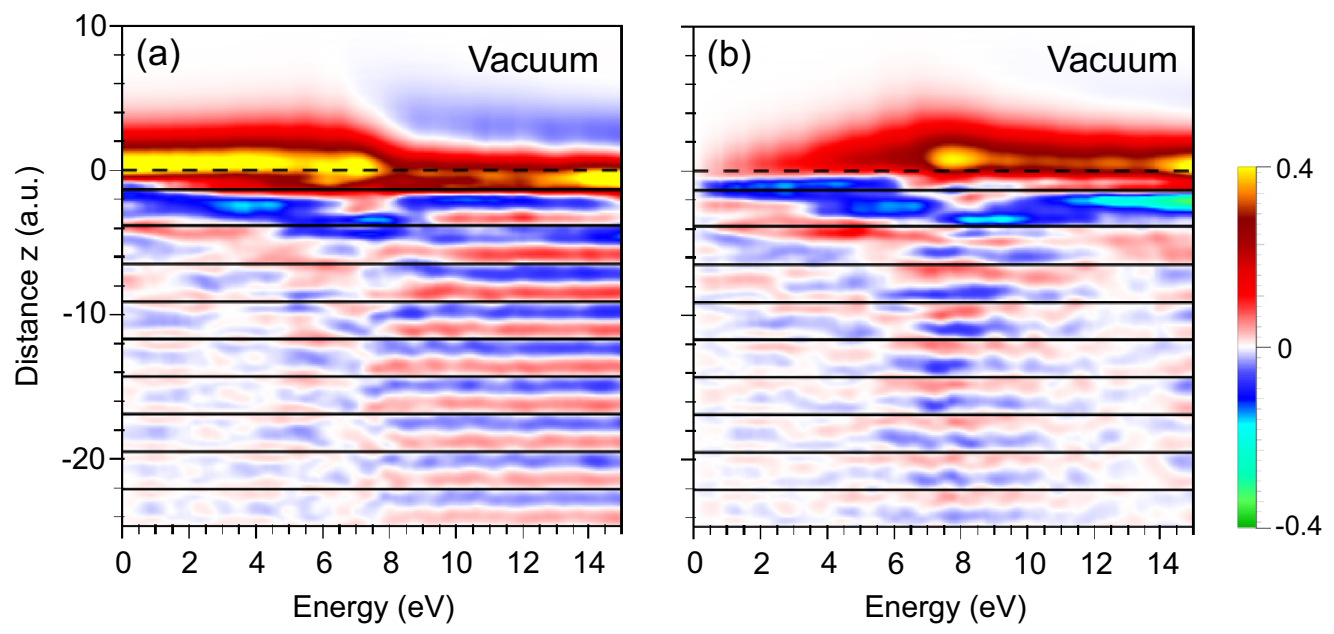

FIG. 5. Distribution of the (a) real and (b) imaginary parts of the ALDA dynamical charge density, $n^{\text {ind }}\left(z, \mathbf{q}_{\|}, \omega\right)$, induced at the Pd(110) surface by an external potential $V^{\text {ext }}(\mathbf{r}, t)$. Results are plotted as a function of energy $\omega$ and coordinate $z$ for $q_{\|}=0.084 \AA^{-1}$ along the $\overline{\Gamma X}$ symmetry direction. The origin of the $z$ coordinate is at the geometrical surface position shown by the horizontal dashed lines. The horizontal solid lines define the palladium atomic layer positions.

external electric field, is substantially reduced in comparison to that in Fig. 5. The excitation of the electronic system inside the bulk becomes less efficient and the confinement of the surface plasmon density to the surface region increases. Further, above the surface atomic layer the peak in $\operatorname{Im}\left[n^{\text {ind }}\left(z, \mathbf{q}_{\|}, \omega\right)\right]$ is clearly visible at energies about $7.6 \mathrm{eV}$, which correlates well with the SP peak identified in the surface loss function.

In our calculations of the surface loss function, we do not find any relevant feature at energies below $\approx 4 \mathrm{eV}$. This discards the evidence of additional surface modes, such as acoustic surface plasmons or low-energy inter-band plasmons. This behavior is possibly due to the large number of surface electronic states in the valence band structure of $\operatorname{Pd}(110)$. The numerous transitions involving such states would not favor the formation of clear resonances corresponding to collective excitations, contrary to the situation in bulk $[45,46]$. Thus the
$\operatorname{Pd}(110)$ surface seems to show a situation where the mere existence of a surface electronic state crossing the Fermi level is not sufficient to create an acousticlike surface mode.

When inspecting the surface loss function in Fig. 3 at small $q_{\|}$'s we find a weak peak at energy about $4.9 \mathrm{eV}$. Since this is observed for $\operatorname{Im}[g]$ we can identify it as a surface-related feature. As seen in Fig. 7, with momentum increase the energy of this peak increases gradually, reducing its spectral weight. After reaching the energy of $5.0 \mathrm{eV}$ at $q_{\|}=0.17 \AA^{-1}$ it disappears. In the experiment of Liu and Brown [41,82] a similar mode was probably detected at energy of $5.2 \mathrm{eV}$ without resolving its dispersion and was interpreted as a surface feature. In the real part of the induced-density distribution of Fig. 5(a) one can distinguish resonating behavior at the energy of $\approx 4.9 \mathrm{eV}$ in the interior of the crystal. $\operatorname{In} \operatorname{Im}\left[n^{\text {ind }}\left(z, \mathbf{q}_{\|}, \omega\right)\right]$, reported in Fig. 5(b), a weak feature oscillating with $z$ can be
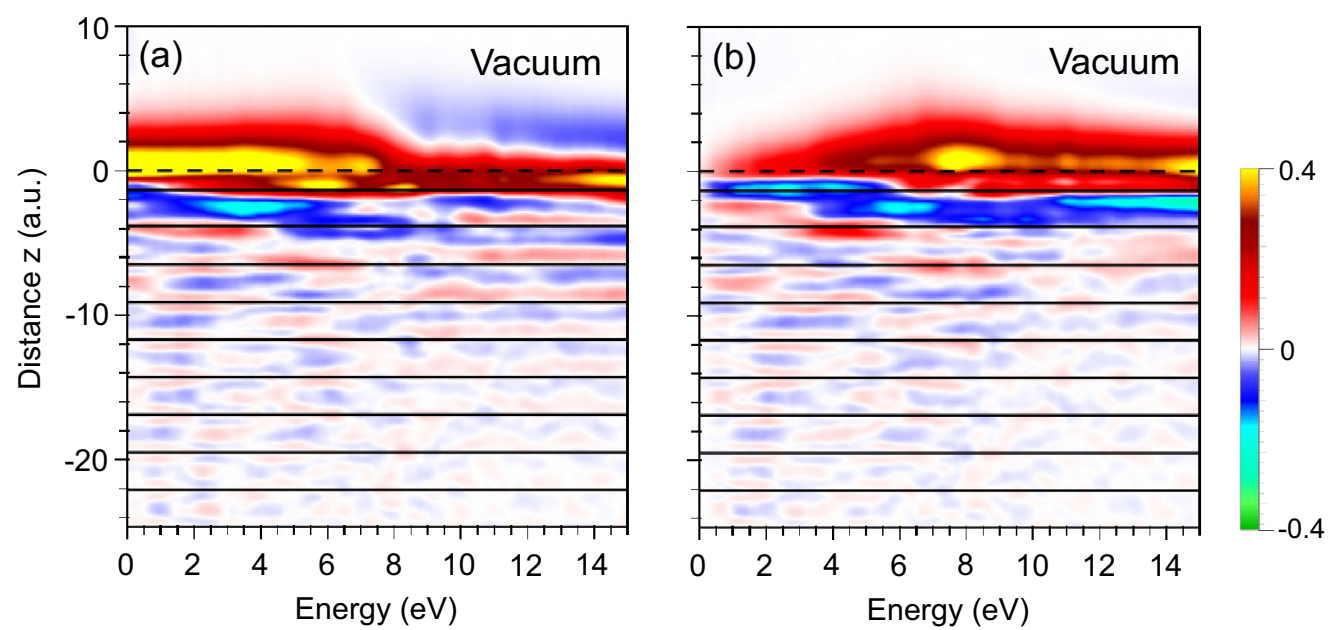

FIG. 6. Distribution of the (a) real and (b) imaginary parts of the ALDA dynamical charge density, $n^{\text {ind }}\left(z, \mathbf{q}_{\|}, \omega\right)$, induced at the Pd(110) surface by an external potential $V^{\text {ext }}(\mathbf{r}, t)$. Results are plotted as a function of energy $\omega$ and coordinate $z$ for $q_{\|}=0.336 \AA^{-1}$ along the $\overline{\Gamma X}$ symmetry direction. The origin of the $z$ coordinate is at the geometrical surface position shown by the horizontal dashed lines. The horizontal solid lines define the palladium atomic layer positions. 


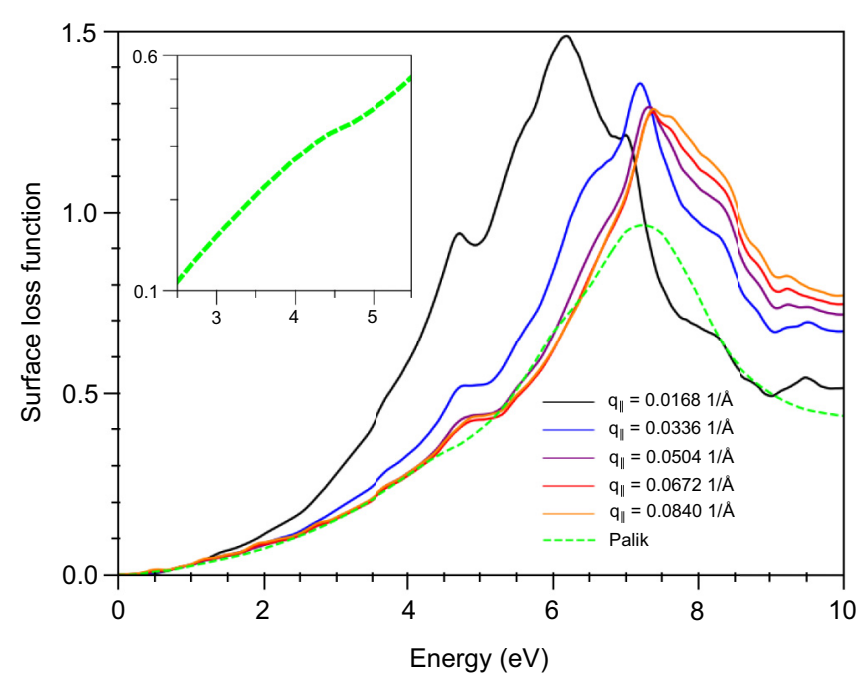

FIG. 7. ALDA surface loss function of $\mathrm{Pd}(110)$ calculated for the indicated momentum transfers. The dashed line shows the surface loss function calculated at $q_{\|}=0$ classically employing the experimental bulk dielectric function of Pd reported in the textbook of Palik [79]. For the latter, the inset shows a zoom in the $2.5-5.5 \mathrm{eV}$ energy interval.

detected at the same energy. This indicates that indeed this feature has origin in the bulk dielectric function of $\mathrm{Pd}$. This is confirmed by the results in Fig. 7 where a weak feature centered at about $4.5 \mathrm{eV}$ can be distinguished in $\operatorname{Im}[g]$ obtained from classical calculations that use the experimental bulk dielectric function of palladium [79].

\section{CONCLUSIONS}

In this paper we have presented the results of $a b$ initio calculations for the excitation spectra of the $\operatorname{Pd}(110)$ surface. At small momentum transfers a prominent peak in the calculated surface loss function corresponding to a conventional surface plasmon is obtained at an energy of $7.35 \mathrm{eV}$. This value is in good agreement with the existing experimental data for $\operatorname{Pd}(110)$ at low $q_{\|}$. We have also found that upon momentum increase, the surface plasmon dispersion presents a weak positive dispersion up to about $q_{\|}=0.4 \AA^{-1}$. However, this finding contradicts the experimental observations of Ref. [43] where strong initial negative surface plasmon dispersion was reported. This may be an example where the $a b$ initio density surface response calculation diverges so strongly with the energy-loss experiment, and more experimental and theoretical work is needed to reveal the origin of such disagreement. From the theoretical side, a description of the collective electronic excitations on this surface would perhaps require to go beyond the dipole approximation based on the use of a surface loss function [5]. In this respect, probably the kinematics of the incident probing electron should be taken into account [83-85].

Our calculation of the surface loss function only shows a strong response at ultraviolet energies, without any signature of low-energy collective excitations in spite of the presence of numerous surface electronic states around the Fermi level on this surface. It seems that a large number of such states at the $\operatorname{Pd}(110)$ surface does not favor the creation of well-defined low-energy surface collective excitations.

The raw data for some representative $q_{\|}$points can be extracted from the metadata deposited at [86].

\section{ACKNOWLEDGMENTS}

We acknowledge funding from the Project of the Basque Government for consolidated groups of the Basque University, through the Department of Universities (Q-NANOFOT IT1164-19) and Project No. PI2017-30 of the Departamento de Educación, Política Linguística y Cultura of the Basque Government. U.M., R.E., and J.A. acknowledge support from the Spanish Ministry of Science and Innovation (Grant No. PID2019-107432GB-I00). V.M.S. acknowledges support from the Spanish Ministry of Science and Innovation (Grant No. PID2019-105488GB-I00).
[1] D. Pines and D. Bohm, Phys. Rev. 85, 338 (1952).

[2] D. Pines and P. Nozières, The Theory of Quantum Liquids: Normal Fermi Liquids (W. A. Benjamin, New York, 1966), Vol. 1.

[3] R. H. Ritchie, Phys. Rev. 106, 874 (1957).

[4] P. J. Feibelman, Prog. Surf. Sci. 12, 287 (1982).

[5] A. Liebsch, Electronic Excitations at Metal Surfaces (Plenum, New York, 1997).

[6] M. Rocca, Surf. Sci. Rep. 22, 1 (1995).

[7] W. Plummer, K.-D. Tsuei, and B.-O. Kim, Nucl. Instrum. Meth. Phys. Res. B 96, 448 (1995).

[8] E. Prodan, C. Radloff, H. J. Halas, and P. Nordlander, Science 302, 419 (2003).

[9] J. M. Pitarke, V. M. Silkin, E. V. Chulkov, and P. M. Echenique, Rep. Prog. Phys. 70, 1 (2007).

[10] M. Dąbrowski, Y. Dai, and H. Petek, Chem. Rev. 120, 6247 (2020).
[11] K.-D. Tsuei, E. W. Plummer, and P. J. Feibelman, Phys. Rev. Lett. 63, 2256 (1989).

[12] K.-D. Tsuei, E. W. Plummer, A. Liebsch, E. Pehlke, K. Kempa, and P. Bakshi, Surf. Sci. 247, 302 (1991).

[13] M. Rocca and U. Valbusa, Phys. Rev. Lett. 64, 2398 (1990).

[14] M. Rocca, F. Biggio, and U. Valbusa, Phys. Rev. B 42, 2835 (1990).

[15] M. Rocca, L. Yibing, F. Buatier de Mongeot, and U. Valbusa, Phys. Rev. B 52, 14947 (1995).

[16] M. Rocca, M. Lazzarino, and U. Valbusa, Phys. Rev. Lett. 69, 2122 (1992).

[17] F. Moresco, M. Rocca, V. Zialasek, T. Hildebrandt, and M. Henzler, Surf. Sci. 388, 1 (1997).

[18] G. Lee, P. T. Sprunger, and E. W. Plummer, Surf. Sci. 286, L547 (1993).

[19] P. J. Feibelman, Surf. Sci. 282, 129 (1993).

[20] A. Liebsch, Phys. Rev. Lett. 71, 145 (1993). 
[21] P. J. Feibelman, Phys. Rev. Lett. 72, 788 (1994).

[22] A. Liebsch, Phys. Rev. Lett. 72, 789 (1994).

[23] V. M. Silkin, P. Lazić, N. Došlić, H. Petek, and B. Gumhalter, Phys. Rev. B 92, 155405 (2015).

[24] V. M. Silkin, E. V. Chulkov, and P. M. Echenique, Phys. Rev. Lett. 93, 176801 (2004).

[25] J. Yan, K. W. Jacobsen, and K. S. Thygesen, Phys. Rev. B 84, 235430 (2011).

[26] J. Yan, K. W. Jacobsen, and K. S. Thygesen, Phys. Rev. B 86, 241404(R) (2012).

[27] A. Politano and G. Chiarello, Prog. Surf. Sci. 90, 144 (2015).

[28] A. Politano, V. Formoso, and G. Chiarello, Plasmonics 3, 165 (2008).

[29] S. J. Park and R. E. Palmer, Phys. Rev. Lett. 102, 216805 (2009).

[30] L. Vattuone, M. Smerieri, T. Langer, C. Tegenkamp, H. Pfnür, V. M. Silkin, E. V. Chulkov, P. M. Echenique, and M. Rocca, Phys. Rev. Lett. 110, 127405 (2013).

[31] S. D. Bader, J. M. Blakely, M. B. Brodsky, R. J. Friddle, and R. L. Panosh, Surf. Sci. 74, 405 (1978).

[32] V. D. Vankar and R. W. Vook, Surf. Sci. 131, 463 (1983).

[33] J. L. Robins, Proc. Phys. Soc. (London) 78, 1177 (1961).

[34] V. V. Zashkvara, M. I. Korshunskii, and V. S. Red'kin, Sov. Phys. Solid State 12, 993 (1970).

[35] K. Okuno, T. Ito, M. Iwami, and A. Hiraki, Solid State Commun. 44, 209 (1982).

[36] H. A. E. Hagelin, J. F. Weaver, G. B. Hoflund, and G. N. Salaita, J. Electron Spectrosc. Relat. Phenom. 124, 1 (2002).

[37] H. A. E. Hagelin-Weaver, J. F. Weaver, G. B. Hoflund, and G. N. Salaita, J. Alloys Compounds 393, 93 (2005).

[38] M. Nishijima, M. Jo, Y. Kuwahara, and M. Onchi, Solid State Commun. 58, 75 (1986).

[39] P. Staib and K. Ulmer, Z. Phys. 219, 381 (1969).

[40] T. Bornemann, J. Eickmans, and A. Otto, Solid State Commun. 65, 381 (1988).

[41] D. R. Liu, Ultramicroscopy 29, 44 (1989).

[42] F. P. Netzer and M. M. El Gomani, Surf. Sci. 124, 26 (1983).

[43] M. Rocca, S. Lizzit, B. Brena, G. Cautero, G. Comelli, and G. Paolucci, J. Phys.: Condens. Matter 7, L611 (1995).

[44] M. Rocca and F. Moresco, Prog. Surf. Sci. 53, 331 (1996).

[45] V. M. Silkin, I. P. Chernov, Y. M. Koroteev, and E. V. Chulkov, Phys. Rev. B 80, 245114 (2009).

[46] V. M. Silkin, V. U. Nazarov, A. Balassis, I. P. Chernov, and E. V. Chulkov, Phys. Rev. B 94, 165122 (2016).

[47] N. V. Smith, C. T. Chen, J. M. Tranquada, and P. D. Johnson, Phys. Rev. B 38, 12259 (1988).

[48] W. Dong, V. Ledentu, P. Sautet, A. Eichler, and J. Hafner, Surf. Sci. 411, 123 (1998).

[49] N. Memmel, G. Rangelov, and E. Bertel, Prog. Surf. Sci. 74, 239 (2003).

[50] V. M. Silkin, A. García-Lekue, J. M. Pitarke, E. V. Chulkov, E. Zaremba, and P. M. Echenique, Europhys. Lett. 66, 260 (2004).

[51] B. Diaconescu, K. Pohl, L. Vattuone, L. Savio, P. Hofmann, V. M. Silkin, J. M. Pitarke, E. V. Chulkov, P. M. Echenique, D. Farías, and M. Rocca, Nature (London) 448, 57 (2007).

[52] S. J. Park and R. E. Palmer, Phys. Rev. Lett. 105, 016801 (2010).

[53] K. Pohl, B. Diaconescu, G. Vircelli, L. Vattuone, V. M. Silkin, E. V. Chulkov, P. M. Echenique, and M. Rocca, Europhys. Lett. 90, 57006 (2010).
[54] T. Langer, D. F. Förster, C. Busse, T. Michely, H. Pfnür, and C. Tegenkamp, New J. Phys. 13, 053006 (2011).

[55] J. Pischel, E. Welsch, O. Skibbe, and A. Pucci, J. Phys. Chem. C 117, 26964 (2013).

[56] X. Jia, S. Zhang, R. Sankar, F. C. Chou, W. H. Wang, K. Kempa, E. W. Plummer, J. Zhang, X. Zgu, and J. Guo, Phys. Rev. Lett. 119, 136805 (2017).

[57] A. Politano, H. K. Yu, D. Farías, and G. Chiarello, Phys. Rev. B 97, 035414 (2018).

[58] V. Despoja, I. Radović, A. Politano, and Z. L. Mišković, Nanomaterials 10, 703 (2020).

[59] V. U. Nazarov, New J. Phys. 17, 073018 (2015).

[60] B. N. J. Persson and E. Zaremba, Phys. Rev. B 31, 1863 (1985).

[61] E. Runge and E. K. U. Gross, Phys. Rev. Lett. 52, 997 (1984).

[62] M. Petersilka, U. J. Gossmann, and E. K. U. Gross, Phys. Rev. Lett. 76, 1212 (1996).

[63] T. Nagao, T. Hildebrandt, M. Henzler, and S. Hasegawa, Phys. Rev. Lett. 86, 5747 (2001).

[64] S. Botti, A. Schindlmayr, R. Del Sole, and L. Reining, Rep. Prog. Phys. 70, 357 (2007).

[65] B.-O. Kim, G. Lee, E. W. Plummer, P. A. Dowben, and A. Liebsch, Phys. Rev. B 52, 6057 (1995).

[66] H. Ishida and A. Liebsch, Phys. Rev. B 54, 14127 (1996).

[67] C. Kittel, Introduction to Solid State Physics, 7th ed. (Wiley, New York, 1996).

[68] M. Skottke, R. J. Behm, G. Ertl, V. Penka, and W. Moritz, J. Chem. Phys. 87, 6191 (1987).

[69] V. M. Silkin, E. V. Chulkov, I. Y. Sklyadneva, and V. E. Panin, Izv. Vyssh. Uchebn. Zaved., Fiz. 27, 56 (1984).

[70] N. Troullier and J. L. Martins, Phys. Rev. B 43, 1993 (1991).

[71] D. M. Ceperley and B. J. Alder, Phys. Rev. Lett. 45, 566 (1980).

[72] J. P. Echeverry, E. V. Chulkov, P. M. Echenique, and V. M. Silkin, Phys. Rev. B 100, 115137 (2019).

[73] V. M. Silkin, I. P. Chernov, P. M. Echenique, Y. M. Koroteev, and E. V. Chulkov, Phys. Rev. B 76, 245105 (2007).

[74] V. M. Silkin, E. V. Chulkov, and P. M. Echenique, Phys. Rev. B 68, 205106 (2003).

[75] J. M. Pitarke, V. U. Nazarov, V. M. Silkin, E. V. Chulkov, E. Zaremba, and P. M. Echenique, Phys. Rev. B 70, 205403 (2004).

[76] V. M. Silkin, R. Díez Muiño, I. P. Chernov, E. V. Chulkov, and P. M. Echenique, J. Phys.: Condens. Matter 24, 104021 (2012).

[77] M. A. Poyli, V. M. Silkin, I. P. Chernov, P. M. Echenique, R. Díez Muiño, and J. Aizpurua, J. Phys. Chem. Lett. 3, 2556 (2012).

[78] X. Zubizarreta, E. V. Chulkov, I. P. Chernov, A. S. Vasenko, I. Aldazabal, and V. M. Silkin, Phys. Rev. B 95, 235405 (2017).

[79] Edited by E. D. Palik, Handbook of Optical Constants of Solids (Academic Press, New York, 1997).

[80] M. Cazzaniga, H.-C. Weissker, S. Huotari, T. Rylkkänen, P. Salvestrini, G. Monaco, G. Onida, and L. Reining, Phys. Rev. B 84, 075109 (2011).

[81] V. Olevano, M. Palumno, G. Onida, and R. Del Sole, Phys. Rev. B 60, 14224 (1999).

[82] D.-R. Liu and L. M. Brown, Acta Metall. 36, 2597 (1988).

[83] V. U. Nazarov, Phys. Rev. B 59, 9866 (1999).

[84] V. U. Nazarov, V. M. Silkin, and E. E. Krasovskii, Phys. Rev. B 93, 035403 (2016).

[85] H.-Y. Deng, Ann. Phys. 418, 168204 (2020).

[86] https://digital.csic.es. 